\title{
Species-specific structural stability of fish myosin
}

KUNIHIKOKONNO

Graduate School of Fisheries Sciences, Hokkaido University, Hakodate, Hokkaido 041-8611, Japan (konno

@fish.hokudai.ac.jp)

SUMMARY: This presentation is a review of the development in the fish muscle protein research, especially myosin. Species-specificity of fish myosin was classified into the following four categories. 1) Structural stability of fish myosin as studied by the denaturation caused by heating, freezing, urea etc. Thermal stability of fish myosin deeply dependent on the environmental temperature is discussed. 2) Structural stability of myosin filaments as investigated by the actin-activated Mg-ATPase activity. 3) Myosin stabilization upon F-actin binding in connection with the accelerated denaturation by salt and pyrophosphate (PPi). Myosin denaturation mode in myofibrils was also discussed. 4) Light chain (LC) binding and its effect on myosin denaturation.

\section{KEYWORDS : myosin, ATPase, denaturation, heating, subfragment-1, rod, filament, acclimation}

\section{INTRODUCTION}

Fish as food, the most important portion would be muscle as protein resources. Myofibrillar proteins occupies the largest quantity of about $50-70 \%$ of total protein. Myosin, as a single protein, occupies about a half of it. Myosin molecule is composed of unique two structures; water soluble head termed subfragment-1 (S-1), in which ATP hydrolysis and actin binding sites locate, and rest of the structure of salt-soluble, a-helical long flexible tail termed rod in which myosin assembles to form filaments. To the very end of S-1 heavy chain (HC), two types of light chain (LC) components associate. Connecting S-1 and rod constructs the neck structure. Rod portion is composed of two portions; water soluble subfragment- 2 and salt soluble light meromyosin (LMM). The former supports the projected head from the thick filaments. The latter is a filament forming domain of myosin molecule. Myosin in myofibrils is in a filamentous form. Once myofibrils are dissolved in high-salt such as $0.5 \mathrm{M} \mathrm{NaCl}$, monomeric myosin attached to long F-actin filament to form arrow-head structure. Accordingly, "denaturation" is not a simple matter for myosin. Studies with myosin fragments or domains, ones with myosin, and ones with myofibrils or actomyosin are needed for a full understanding of "myosin denaturation" or "myosin stability".

Research on fish myosin started as early as 1950 's, a very similar year when study on mammalian myosin research started. Fish myosin isolation was first recorded by Connell from Atlantic cod in 1954, 1) and his series of paper showed the unstable nature of cod myosin readily forming aggregates during the storage in ice?) This would be the first mention of instability of fish myosin. His work was inherited to many Japanese muscle researchers, and many reports on the isolation, biochemical and physicachemical characterization of fish myosin have been accumulated. Study on the instability of fish myosin is still one of the major subjects. For examples, how fish myosin adapts to the low temperature, what are structural bases in the unstable nature of fish myosin, how the unstable nature of fish myosin is overcome, how the unstable nature affects the properties of products are in progress.

I reviewed the species-specific stability of fish myosin since Connell's study.

\section{1) Structural stability \\ Thermal stability}

There are many factors to induce structural changes or denaturation of myosin. The first and prominent one would be thermal treatment, and is most extensively investigated. In 1960, Connell mentioned the unstable nature of cod myosin by finding a rapid aggregates formation analyzed by ultracentrifugal sedimentation technique. 2) Buttkus with trout myosin confirmed it 3) by employing ATPase inactivation for detecting fish myosin denaturation. The first record of the isolation of fish myosin in Japan was done by Takashi in 1970 with carp, 4) and firstly showed its purity by SDS-polyacrylamide gel electrophoresis (PAGE). 5) By using the preparative method developed by Connell, myosin from many species were isolated; tuna, 6) 
yellowtail, 7) mackerel, 8) walleye pollack, 9) and shark. 10) All reports claimed similar physicochemical and biochemical properties of fish myosin to those of rabbit myosin except instability.

Indeed, preparative method of myosin was established, the method is still troublesome, and myosin isolated cannot be stored because of the unstable nature. To overcome the problem, myofibrils were introduced by Katoh. et al. 11) as a useful material for study myosin stability.

In 1973, important proposal was made separately by Jonston et al.12) and by Arai et al.13) that thermal stability of myofibrils or actomyosin as measured by Ca-ATPase inactivation is species-specific and deeply related to their body or habitat temperatures. Hashimoto et al. ${ }^{14)}$ clearly demonstrated the correlation between the activation energy for myosin denaturation and habitat water temperature. To be functional, myosin molecule should have a similar flexibility at the individual habitat temperature indicating that myosin structure from cold water species is less rigid than that from warm water species. It is natural that less rigid myosin from cold-water species is much less unstable than that from warm water species when compared at the specific temperature. Studies on the thermal denaturation of actomyosin reconstituted from myosin and actin of various species of fish, scallop and rabbit clearly demonstrated the myosin species dependent thermal stability. ${ }^{15}$ )

To elucidate how fish adapts to the change in the water temperature, Watabe's group enthusiastically studied by using carp which survives at wide range of temperature. 16-18) They introduced biotechnological and genetic techniques to the study. They found thermal stability of myosin changes by acclimation to the temperatures of 10 and $30^{\circ} \mathrm{C}$, and concluded that carp acclimated to 10 and $30^{\circ} \mathrm{C}$ by expressing different isomer with different stability. 19) They sequenced the whole myosin heavy chain ( $\mathrm{HC}$ ) of the isomers to analyze which residues are involved in the different stability. Mutation is a promising one, but further studies are needed for its complete understanding.

Myosin was consisted of two completely different structures, subfragment-1 (S-1, head) and rod (tail). Myosin molecule is cleaved into these two by digesting with chymotrypsin or papain. The first S-1 preparation was reported from carp by Ikariya et al. in 1981. 20) Studies on the thermal inactivation of fish S-1 confirmed the previous proposal of species-specific stability of fish myosin.

Rod portion has an ability to assemble to form filaments. Reports on the thermal stability of rod portion were limited. Habitat temperature dependent structural stability of rod was reported by King $e l$ al. 21) Kato el al. 22) claimed that alcohol treatment, which is conventionally used for the removal of contaminated myosin from rod preparation, causes a marked aggregation in carp myosin rod. They developed a new isolation method of monomeric rod for the study of filament forming ability of fosh myosin. Rod prepared from the acclimated carp to 10 and $30^{\circ} \mathrm{C}$ had temperature dependent stabilities as revealed by unfolding analysis using differential scanning calorimetry apparatus. 23 ) The same conclusion was withdrawn with LMM. 24,25)

Separate experiments with S-1 and rod showed a habitat temperature dependent thermal stability. However, there was no information how S-1 and rod portion interacts in the thermal denaturation process of myosin. A peculiar phenomenon that myosin forms oligomers before loosing ATPase activity was reported. 26) Chymotryptic digestion analysis of the oligomers formed and denaturation analysis of the digest upon heating suggested a new role of neck region in myosin structural changes. Active oligomers were visualized by electron microscopic observation as ones containing myosin molecule with double-headed structure. Head structure for the inactive oligomers were clearly distinguishable from active one showing fused head structure (Tazawa T \& Konno K, unpubl. data, 1999).

\section{Freezing stability}

Second effector would be freezing. Frozen storage of fish is an excellent way to maintain the freshness. Many researches on the freeze denaturation of fish muscle protein have done. A recent study on freeze denaturation of carp S-1 showed that S-1 underwent less severe damage during a frozen storage with a slow aggregation. 27) This was basically true for myofibrils. 28)

Unstable nature of fish myosin encouraged us to find stabilizing compounds as food additives. The most prominent ones and most widely used would be sugar (or sugar alcohol)29) Stabilizing effect of sugars is concentration dependent and effectiveness was determined by the numbers of hydroxyl group in the structure. ${ }^{30)} \mathrm{Na}$-glutamate also strongly suppresses the thermal and freeze denaturation. ${ }^{31}$ ) One of the most unstable fish species is would be walleye pollack. The isolation of pollack myosin required the inclusion of high concentrations of sorbitol or Na-glutamate in the buffer for preventing the denaturation. 9,32) Fish 
myosin could be directly prepared from fish muscle as Connell first reported. However, much easier method using myofibrils as the starting material was developed. The method contains salting-out with ammonium sulfate in the presence of Mg-ATP. 33) Salting-out is old-fashioned, but the method is still useful for the isolation of unstable myosin because sulfate very strongly stabilizes myosin. ${ }^{34)}$

\section{Stability to other denaturations}

Shark muscle contains urea as high as $0.4 \mathrm{M}$. More resistant structure of shark myosin than carp myosin against urea treatment was demonstrated by measuring actin-activated $\mathrm{mg}$-ATPase in the presence of various concentrations of urea. ${ }^{10)}$

Oxidation would be another denaturing factor. Myosin contains reactive SH-groups both in S-1 and rod regions. Oxidation of the most reactive $\mathrm{SH}$ group in $\mathrm{S}-1$, termed $\mathrm{SH} 1$, accelerated fish myosin denaturation by $2-4$ fold. ${ }^{35}$ ) SS bonding catalyzed by a trace amount of copper ion formed dimers with myosin 36,37 ) and rod. 38) Fish lipid is rich in polyunsaturated fatty acids that are readily oxidized by oxygen in air. Incubation of myosin with the oxidized triglyceride altered the ATPase activity and induced the cross-linking of myosin through oxidation of SH groups. ${ }^{39}$ )

\section{2) Filament formation}

Myosin associates to form filaments in a physiological ionic strength. Myosin as filaments interacts with F-actin filament leading to a contraction which is accompanied by an accelerated ATP hydrolysis, actin-activated $\mathrm{Mg}$-ATPase. Actin activation profile of myosin Mg-ATPase of fish was found to differ from species to species. Carp was a typical species whose myosin was strongly activated at low concentrations of F-actin exhibiting a saturated activation profile. Tilapia is an opposite one whose myosin was very weakly activated. Its activation profile rather resembled that with water-soluble S-1.40) These difference was proved to come from different filament forming ability of two species of myosin. Myosin that formed long and stable filaments was strongly activated, and myosin that formed short and fragile filament was weakly activated.

The shortest filament forming domain of myosin was isolated as the carboxyl terminal fragment of rod with a size of the $40 \mathrm{kDa} .41$ ) Corresponding fragment was also isolated from tilapia, and its filament forming ability was compared with that of carp fragment. It was demonstrated that forming ability of the fragment from tilapia was intrinsically less than that of carp fragment. 42)

Filament forming ability of myosin reflected unique changes in the actin-activated Mg-ATPase upon heating of myosin. Upon heating carp myosin, Ca-ATPase inactivation proceeded slowly, while actinactivated Mg-ATPase dropped very quickly. ${ }^{43}$ ) On the other hand, Mg-ATPase activity for tilapia myosin rather increased upon heating. 44) These opposite effects of heating on carp and tilapia was well accounted for by the different effect of heating on the filament forming ability of two species of myosin. Heating damaged the filament forming ability of carp myosin, while improved the ability of tilapia myosin revealed by electron microscopic observation of the filaments formed.

Another filament formation related unique event was a significant enhancement of Mg-ATPase activity observed when myofibrils were heated. 45) This activation was explained by an altered filament structure accompanied by a loss of salt solubility. Insolubilization of myosin filaments would lead to increase the possibility of attaching myosin head to F-actin, essential step for activation.

\section{3) F-actin binding and stability}

It is established that F-actin strongly stabilizes myosin upon binding. Generally, F-actin is much more stable than myosin explaining that the thermal stabilities of reconstituted actomyosin are all myosin species dependent. However, a very important finding was made by Wakameda et al. that F-actin in myofibrils is much more labile than myosin when exposed to high-salt. Wakameda et al ${ }^{46)}$ extensively studied how salt concentration affects the thermal inactivation of myofibrils. Their first finding was that ATPase inactivation rate $\left(\mathrm{K}_{\mathrm{D}}\right)$ for myofibrils gradually increased with increasing salt concentration, and reached to the maximal level at around $1-2 \mathrm{M} \mathrm{NaCl}$. The maximal $\mathrm{K}_{\mathrm{D}}$ for myofibrils there was the same as that for myosin alone indicating that myosin in myofibrils lost the protection by F-actin under such conditions. This was accounted for by a selective denaturation of F-actin at high concentrations of salt. 47) The second finding was that salt concentration required for a loss of protection by F-actin was dependent on the species of fish. Moreover, an addition of pyrophosphate (PPi) lowered the salt concentration for F-actin denaturation. 48) When the medium contained $\sim \mathrm{mM}$ PPi, $0.5 \mathrm{M}$ 
$\mathrm{NaCl}$ was high enough to cause $\mathrm{F}$-actin denaturation indicating that $\mathrm{F}$-actin was protected by myosin from salt denaturation. This would be the first statement of the mutual stabilization of F-actin and myosin. The another finding was that the extent of stabilization by F-actin achieved in low-salt medium depended on the species examined. Among fish species examined, white croaker myosin was most strongly stabilized, about 250 times. Cod myosin showed the smallest stabilization of about only 15 times. There was no correlation between the magnitude of the stabilization by F-actin and the specific thermal stability. This different extent of stabilization by F-actin was proved to be determined by myosin species by the hybridization experiments (Takahashi $\mathrm{M}$ \& Konno $\mathrm{K}$, unpubl. data, 1999). Carp and walleye pollack were selected as species with large and small extent of stabilization by F-actin, respectively, Myosin in walleye pollack myofibrils was cleaved into S- 1 and rod, then pollack S-1 was replaced by carp S-1 separately isolated in the presence of Mg-ATP. The hybrid specimen gave exactly the same stabilization as carp myofibrils did. Further studies are required what are the differences in the two species of S-1.

Very recently, an important finding was made. 49) When carp myofibrils were heated, thermal denaturation of S-1 and rod portion proceeded differently. S-1 and rod denaturation profiles were further investigated for myofibrils from other species of fish. There was a great diversity in the S-1, rod denaturation profiles. Although rod portion is much stable than $S-1$ in a separated state, rod denaturation in carp and rainbow trout myofibrils was much faster than S-1 portion. On the other hand, rod denaturation in walleye pollack myofibrils was slower than S-1. When relative denaturation rate of rod to that of S-1 was referred to as the indicator for characterizing the denaturation mode, a close correlation was observed between the ratio and the extent of stabilization by F-actin in low-salt medium (Takahashi M \& Konno K, unpubl. data, 1999). Therefore, S-1 portion determines not only the magnitude of stabilization by F-actin but also determined the myosin denaturation mode.

This specificity in the denaturation mode of S-1 and rod disappeared when myofibrils were heated in the medium containing $\mathrm{KCl}$ above $0.3 \mathrm{M}$, heated as actomyosin. A quick loss of solubility when heated in $0.1 \mathrm{M} \mathrm{KCl}$ was no longer detected for the dissolved myofibrils for all species of myofibrils examined. However, chymotryptic digestion of the heated myofibrils showed a production of large quantity of aggregated rod. Aggregation profile was almost the same as the S-1 denaturation profile. As isolated rod never formed aggregates under the same conditions, it is certain that rod aggregation proceeded S-1 denaturation as a determinant step. In the dissolved myofibrils, myosin is attached to F-actin at its S-1 region with a monomeric tail. As long F-actin filament has great mass compared with that of monomer myosin, thermal energy applied would cause a movement of myosin tail. The movement would induce tangling of myosin molecule, especially in rod portion. When S-1 portion denatured in this form, aggregation at S-1 readily took place, and this subsequently induced rod aggregation (Takahashi M \& Konno K, unpubl, data, 1999).

\section{4) ATP and PPi binding and stability}

It is well known that myosin that bound ATP, substrate, is very stable. Analogous compounds such as $\mathrm{ADP}$ or $\mathrm{PPi}$ also stabilize myosin with less magnitude. ATP equally stabilized myosin from several species of fish, while stabilizing effect of PPi differed from species to species. PPi stabilized S-1 from carp similarly to ADP in low-salt, while accelerated the inactivation at high-salt where ADP still stabilized S-1. Tilapia S-1 was destabilized at all salt concentration upon PPi addition. Thus, PPi affects the denaturation of fish myosin in two ways; destabilization by removing the protection by F-actin through dissociation, and a direct destabilization of myosin upon binding.

\section{5) Light chain and stability}

Myosin contained two types of LC components, regulatory or Ca-binding $\mathrm{LC}$, and essential or alkali LC. Different LC composition in fish myosin was first recorded by Takashi with tilapia myosin 50 . Species-specific LC composition of fish myosin was well applied as an indicator for the identification of fish species. 51,52 ) Chymotryptic $\$-1$ preparation usually contains two alkali LC with different size. By separating these two isozymes, involvement of LC component in the stability of S-1 isozyme was demonstrated. ${ }^{53)}$

Effect of LC binding on the denaturation mode of S-1 was also studied. Thermal inactivation, aggregate formation, and LC dissociation was compared among S-1 from several species of fish and rabbit. S-1 from carp readily dissociated $\mathrm{LC}$ components immediately after inactivation leading to a turbid aggregate 
formation by naked S-1 HC. LC dissociation for tilapia S-1 was less, and rabbit S-1 dissociated practically no LC even after inactivation showing no turbid aggregate formation. 54) LC components was found to affect the denaturation mode of fish S-1

\section{REFERENCES}

1. Connell JJ. Studies on the proteins of fish skeletal muscle. 3. Cod myosin and actinBiochem. J.1954; 58 : 360-367.

2. Connell JJ. Studies on the proteins of fish skeletal muscle. 7. Denaturation and aggregation of cod myosin. Biochem. J960; 75 : 530-538.

3. Buttkus $H$. Preparation and properties of trout myosin. J. Fish. Res. Boad. Canada 1966; 2 3: 563-573.

4. Takashi R, Arai K, Saito T. Studies on muscular proteins of fish. I. Preparation of myosin from carp muscle. Nippon Suisan Gakkaishi1970; 36 : 165-168.

5. Takashi T, Murozuka T , Arai K. Purification and properties of tilapia myosin from dorsal muscle. Nippon Suisan Gakkaishi 1974;40: 1155-1161.

6. Murozuka T, Arai K. Purification and thermo-stability of myosin Ca-ATPase from the frozen muscle of yellowfin tuna. Nippon Suisan Gakkaishi 1976;4 2: 65-70.

7. Kimura I, Murozuka T Arai K. Comparative studies on biochemical properties of myosins from frozen muscles of marine fishes. Nippon Suisan Gakkaishi 1977;43 315 321.

8. Watabe $S$, Hashimoto $K$. Myosins from white and dark muscles of mackerel. Some physico-chemical and enzymatic properties.J. Biochem.1980;8 7: 1491-1499.

9. Kimura I, Takahashi M, Nagahisa E , Fujita T. Preparation of monomeric myosin from Alaska pollack frozen surimi. Nippon Suisan Gakkaishi1982; 4 8: 251.

10. Kanoh S, Watabe $\mathbf{S}$, Hashimoto K. Isolation and some physicochemical properties of requiem shark myosin. Nippon Suisan Gakkaishi 1983;4 9: 757-763.

11. Katoh N, Uchiyama H, Tsukamoto S, Arai K A biochemical study of fish myofibrillar ATPase. Nippon Suisan Gakkaishi 1977;43: 857-867.

12. Johnston IA, Freason N, Golberg G. The effect of environmental temperature on the properties of myofibrillar adenosine triphosphatase from various species of fish. Biochem. J.1973;1 33: 735-738.

13. Arai K, Kawamura K, Hayashi C. The relative thermostability of the actomyosin-ATPase from the dorsal muscles of various fish species. Nippon Suisan Gakkaishi 1973; 39: 1077-1085.

14. Hashimoto A, Kobayashi A, Arai K. Thermostability of fish myofibrillar Ca-ATPase and adaptation to environmental temperature.iNippon Suisan Gakkaishi 1982; 48: 671-684.

15. Yamashita $Y$, Arai K, Nishita K. Thermo-stabilities of synthetic actomyosins in various combinations of myosin and actin from fish, scallop, and rabbit muscles. Nippon Suisan Gakkaishi 1978;4 4: 485-489.

16. Hwang GC, Watabe $S$, Hashimoto $\mathrm{K}$. Changes in carp myosin ATPase induced by temperature acclimation. $J$. Comp. Physiol. B1990; 160 : 233-239.

17. Hwang G-C, Ochiai Y, Watabe S, Hashimoto K. Changes of carp myosin subfragment-1 induced by temperature acclimatioromp. Physiol. B 1991; 161 : 141-146.

18. Guo X-F , Watabe S. ATPase activity and thermostability of actomyosins from thermally acclimated carp. Nippon Suisan Gakkaishi 1993; 5 9: 363-369.

19. Imai J, Hirayama Y, Kikuchi K, Kakinuma M, Watabe S. cDNA cloning of myosin heavy chain isoforms from carp fast skeletal muscle and their gene expression associated with temperature.J. Exp. Biol.1997;200: 27 34.

20. Ikariya T, Kimura I , Arai K. Preparation and biochemical properties of subfragment-1 from dorsal muscle of carpCyprinus carpio Nippon Suisan Gakkaishi 1981; 4 7: 947-955.

21. King $L$, Lehrer SS. Thermal unfolding of myosin rod and light meromyosin: Circular dichroism and tryptophan fluorescence studies. Biochemistry 1989; 2 8: 3498 . 3502 .

22. Kato $\mathrm{S}$ Konno $\mathrm{K}$. Isolation of carp myosin rod and its structural stability. Nippon Suisan Gakkaishi 1993; 59 : 539-544.

23. Nakaya $M$, Watabe $S$, Ooi $T$. Differences in the thermal stability of acclimation temperature-associated types of carp myosin and its rod on differential scanning calorimetry. Biochemistry 1995; 3 4: 3114-3120.

24. Kakinuma M, Hatanaka A, Fukushima H, Nakaya M, Maeda K, Doi Y, Ooi T, Watabe S. Differential scanning calorimetry of light meromyosin fragments having various lengths of carp fast muscle isoforms. Biochem.

2000; 1 28: 11-20.

25. Kakinuma M, Funabara D, Nakaya M, Hirayama $Y$, Watabe S, Maeda K, Ooi $\mathrm{T}$. Thermal unfolding of the coldacclimated type of carp light meromyosin expressed by recombinant DNA inEscherichia coliFisheries Sci1997; 6 3: $1008-1013$.

26. Kato S, Koseki H, Konno K. Oligomerization of carp myosin which retains its ATPase activityisheries Sci.

1996; 6 2: 985-989.

27. Azuma Y, Konno K. Freeze denaturation of carp myosin subfragment-1 as compared with thermal denaturation. Fisheries Sci1999; 65 : 455-458.

28. Azuma $Y$, Konno $K$. Freeze denaturation of carp myofibrils compared with thermal denaturation. Fisheries Sci. 1998; 64 : 287-290.

29. Arai K, Takahashi H, Saito T. Studies on muscular proteins of fish III. Inhibition by sorbitol and sucrose on the denaturation of carp actomyosin during frozen storage. Nippon Suisan Gakkaishi1970; 36 : 232-236. 
30. Ooizumi T, Hashimoto A, Ogura J, Arai $K$. Quantitative aspect for protective effect of sugar and sugar alcohol against denaturation of fish myofibrils. Nippon Suisan Gakkaishi1981;47: 901-908.

31. Noguchi S, Matsumoto JJ. Studies on the control of the denaturation of fish muscle proteins during the frozen storage I. Preventive effect of Na-glutamate. Nippon Suisan Gakkaishi 1970;3 6: 1078-1087.

32. Konno K. Suppression of thermal denaturation of myosin subfragment-1 of Alaska pollack (Theragra chalcogrammaby sorbitol and accelerated inactivation by pyrophosphate. J. Food Sci.1992;5 7: 261-264.

33. Koseki H, Kato S, Konno K. Myosin extractability as a sensitive probe for the thermal denaturation of carp myofibrils. Nippon Suisan Gakkaishi1993;59: 515-518. 34. Konno K. Suppressive effect of sulfate on fish myosin denaturation. Fisheries Sci.1998;6 4: 848-849.

35. Murozuka $T$. Comparative studies on the enzymic properties and thermal stabilities of $\mathrm{N}$-ethylmaleimide modified myosins from fish dorsal and rabbit skeletal muscles Fisheries Sci.1979;45: 1503-1512.

36. Itoh $\mathrm{Y}$, Yoshinaka $\mathrm{R}$, Ikeda $\mathrm{S}$. Behavior of the sulfhydryl groups of carp actomyosin by heating. Nippon Suisan Gakkaishi1979;4 5: 1019-1022.

37. Kishi $A$, Itoh $Y$, Obatake $A$. The polymerization of protein through disulfide bonding during the heating of carp myosin. Nippon Suisan Gakkaishi 1995;6 1: 75-80.

38. Sompongse $W$, Itoh $Y$, Obatake $A$. Responsibility of myosin rod for the dimer formation of myosin heavy chain through SS bonding during ice storage of carp actomyosin. Fisheries Sci1996; 6 2: 473-477.

39. Kawasaki K, Ooizumi $T$, Konno $K$. Effect of peroxidized fish lipid on the ATPase activity of carp myofibrils. Nippon Suisan Gakkaishi 1991; 5 7: 11851191 .

40. Matsuura $M, K o n n o ~ K$, Arai $K$. Thick filaments of fish myosin and its actin-activated $\mathrm{Mg}$-ATPase activity. $C o m p$. Biochem. Physiol.1988;90 B: 803-808.

41. Kato $S$, Konno K. Filament-forming domain of carp dorsal myosin rod.J. Biochem. 1993; 11 3: 43-47.

42. Kato $S$, Konno $K$. Substructure of tilapia myosin rod and its filament forming ability.isheries Sci.1996; 62 : 439-443.

43. Kimura I, Arai K, Takahashi K, Watanabe S. Earliest event in the heat denaturation of myosin. J. Biochem. 1980;8 8: $1703-1713$.

44. Matsuura M, Arai K. Filament forming ability of tilapia myosin in association with biochemical activities. Nippon Suisan Gakkaishi1985; 51 : 1697-1704.

45. Konno K, Ueda Y. Mg-ATPase activity enhancement of carp myofibrils upon thermal treatmentNippon Suisan Gakkaishi 1989;5 5: 1457-1462.

46. Wakameda A, Nozawa S, Arai K. Effect of neutral salts on thermal denaturation of myofibrillar Ca-ATPase of fish Nippon Suisan Gakkaishi 1983;49: 237-243.

47. Wakameda A, Arai K. Dissociation of carp myosin B into actin and myosin in the presence of high concentration of NaCl. Nippon Suisan Gakkaishi 1986; 5 2: 293-300.

48. Matsukawa M, Iwami S, Arai K. Comparative study on denaturation of carp and Alaska pollack myosin $\mathrm{B}$ induced by inorganic pyrophosphate. Nippon Suisan Gakkaishi 1990; 5 6: 287-295.

49. Konno K, Yamamoto T, Takahashi M, Kato S. Early structural changes in myosin rod upon heating of carp myofibrils. J. Agric. Food Chem.2000; 4 : 4905-4909.

50. Takashi R, Murozuka T, Arai K. Light chains of myosin from fish dorsal and rabbit skeletal muscles. Nippon Suisan Gakkaishi1974;40: 1063-1069.

51. Watabe $S$, Ochiai $Y$, Hashimoto $K$. Identification of 5,5'-dithio-bis-2-nitrobenzoic acid (DTNB) and alkali light chains of piscine myosin. Nippon Suisan Gakkaishi 1982; 48: 827-832.

52 . Seki N. Identification of fish species by SDSpolyacrylamide gel electrophoresis of the myofibrillar proteins. Nippon Suisan Gakkaishi 1976;42 1169. 1176.

53. Ochiai Y, Handa A, Kobayashi $T$, Watabe $S$, Hashimoto $K$. Isolation and enzymatic properties of myosin subfragment 1 isozymes from the ordinary muscle of tilapia Oreochromis niloticus iNippon Suisan Gakkaishi 1989;5 5: 2143-2149.

54. Hamai M, Konno K. Structural stability of fish myosin subfragment-1Comp. Biochem. Physiol. 1990; 95 B: 225-259. 